\title{
NÍVEIS DE LEVEDURA DESIDRATADA "SPRAY-DRIED" NA DIETADE ALEVINOS REVERTIDOS DE TILÁPIA DO NILO (Oreochromis niloticus L.)
}

\section{LEVELS OF DRIED YEAST SPRAY-DRIED IN DIET FOR REVERSED NILE TILÁPIA FINGERLINGS (Oreochromis niloticus)}

\section{Wilson Massamitu Furuya ${ }^{1}$ Simoni Seron ${ }^{2}$ Lauro Vargas ${ }^{3}$ Carmino Hayashi ${ }^{4}$ Valéria Rossetto Barriviera Furuya $^{5}{ }^{\text {Claudemir Martins Soares }}{ }^{5}$}

RESUMO

Foram utilizados 1200 alevinos revertidos de tilápia do Nilo com peso inicial médio 0,13 $\pm 0,01 \mathrm{~g}$, distribuídos em 20 aquários de $1000 \mathrm{~L}$ para avaliar os efeitos da inclusão de levedura desidratada na dieta. Os animais foram distribuídos em um delineamento inteiramente casualizado com cinco tratamento $(0,00 ; 7,80 ; 15,60 ; 23,40$ e $31,20 \%$ de levedura na dieta) e quatro repetições. Não foi observado efeito dos tratamentos sobre a conversão alimentar e sobrevivência. Foi observado efeito quadrático sobre o ganho de peso $(Y=l, 7133+0,0477 X$ $\left.0,0017 X^{2}\right)$ e custo em ração/kg ganho $(Y=0,6170$ $\left.0,0112 X+0,0004 X^{2}\right)$. Concluiu-se que o melhor nível de inclusão de levedura seca na dieta para alevinos revertidos de tilápias do Nilo é de $14 \%$.

Palavras-chave: alevinos, levedura seca, níveis de inclusão, Oreochromis niloticus, tilápia do Nilo.

\section{SUMMARY}

One hundred and two thousand reversed of Nile tilápia fingerlings (Oreochromis niloticus) with initial weight mean $0.13 \pm 0.01 \mathrm{~g}$, distributed in twenty 1000-liter fish aquarium, were used to evaluate the inclusion of dried yeast in the diet. Samples were distributed in a entirely randomized design with five treatments $(0.00 ; 7.80 ; 15.60 ; 23.40$ and $31.20 \%$ yeast in the diet) andfour replicates. There was no effect of treatments on food conversion and survival. A quadratic effect over weight gam $\left(Y=1.7133+0.0477 X-0.0017 X^{2}\right)$ and cost of diet/kg gain $\left(Y=0.6170-0.0112 X+0.0004 X^{2}\right)$ was observed. It was concluded the best level of dried yeast in diet for reversed Nile tilapia fingerlings is $14 \%$.
Key words: dried yeast, fingerlings, inclusion levels, Oreochromis niloticus, Nile tilapia.

\section{INTRODUÇÃO}

A tilápia do Nilo tem sido amplamente utilizada para a piscicultura em vários países, sendo esse fato atribuído à sua carne de boas características organolépticas e pelo seu filé, que não apresenta espinhas intramusculares em forma de "Y", além da sua rusticidade e precocidade. Atualmente, tem-se preconizado o cultivo somente de machos, para evitar problemas com reprodução nos tanques de engorda e competição pelo alimento. O método mais utilizado para obtenção de populações monossexo é a reversão sexual das larvas, que consiste da adição de hormônio masculinizante na ração.

O Brasil é o maior produtor mundial de álcool de cana-de-açúcar, com uma produção estimada em 15 bilhões de litros na safra de 97/98, estimando-se para esse período uma produção de 300.000 toneladas de levedura (BUTOLO, 1997). Existem diferentes métodos de secagem de levedura. Entre eles, destacase o uso de rolos rotativos e o método "spray dried". O primeiro método consiste na centrifugação da vinhaça após a destilação do vinho, que, posteriormente, é

\footnotetext{
${ }^{1}$ Professor MSc., Departamento de Zootecnia, Universidade Estadual de Maringá (UEM), Av.: Colombo, 5790, 87020-900, Maringá PR. Furuya@wnet.com.br. Autor para correspondência.

${ }^{2}$ Aluna do curso de Graduação em Zootecnia, UEM, Bolsista, PIBIC/CNPq.

${ }^{3}$ Professor, Doutor, Departamento de Zootecnia, UEM.

${ }^{4}$ Professor, Doutor, Departamento de Biologia, UEM.

${ }^{5}$ Doutorando, Pós-Graduação em Ecologia de Ambientes Aquáticos Continentais, UEM. Recebido para publicação em 22.06.99. Aprovado em 10.11.99
} 
desidratada através de rolos rotativos, processo no qual a temperatura pode ser superior a $200^{\circ} \mathrm{C}$ (LANDELL et al., 1994), sendo que pelo método "spray dried" o material é conduzido até uma câmara quente onde o mesmo é aspergido, ocorrendo a secagem pela entrada de ar quente. Nesse último método, o tempo de exposição e a temperatura são inferiores em relação ao método que utiliza rolo rotativo, resultando em um produto final de melhor valor nutritivo (PERRY et al., 1984).

A levedura desidratada (Saccharomyces cerevisiae), além de apresentar um elevado nível proteico, apresenta inda uma proteína de elevada qualidade pelo seu balanceamento em aminoácidos (GHIRALDINI \& ROSSELL, 1997). Ainda que os níveis das vitaminas A e C sejam reduzidos (DESMONTS, 1968), são ricas em vitaminas do complexo B (MIYADA \& LAVORENTI, 1979; KRONKA et al., 1991), destacando-se os níveis de tiamina, riboflavina, niacina e ácido pantotênico. Portanto, esse alimento constitui-se em importante ingrediente como suplemento vitamínico, sendo que estudos têm demonstrado que as vitaminas das leveduras, em quantidade equivalente, possuem efeitos superiores aos obtidos com as vitaminas sintéticas. (BUTOLO, 1997). Os níveis de aminoácidos sulfurados são relativamente baixos em relação ao farelo de soja, sendo que a levedura obtida pelo método "spray dried" é considerada como boa fonte de lisina.

De uma forma geral, RUMSEY et al. (1990) citam que os trabalhos têm demonstrado a possibilidade de inclusão em valores próximos a $10 \%$ para peixes e animais não-ruminantes. Em relação a sua utilização na alimentação de peixes PEZZATO et al. (1982) utilizaram rações isoprotéicas (25\%), para carpa comum (Cyprínus carpia) e observaram que a substituição de $33,3 \%$ de farinha de carne por levedura seca proporcionou melhor desempenho. DAVIES \& WAREHAM (1988) observaram que a inclusão acima de $15 \%$ de levedura para a tilápia (Oreochromis mossambicus) resultou em uma redução progressiva no valor da dieta. Já para alevinos de tilápia do Nilo, ALVES et al. (1988), em experimento objetivando avaliar níveis de $0 ; 33 ; 66$ e 100\% de levedura desidratada de vinhaça (S. cerevisiae), observaram que esse ingrediente pode substituir até $36,97 \%$ do farelo de soja.

Níveis superiores de inclusão $(50 \%)$ foram efetivos na criação da truta do lago (Salvelinus namaycush) (RUMSEY et al. (1990). Mais recentemente, PADUA (1996) observou que o nível de inclusão de levedura de $29,88 \%$ não prejudicou o desempenho produtivo e o metabolismo de pacu (Piaractus mesopotamicus), durante a fase inicial (40 a 120g), sendo que o nível de inclusão de 39,8\% elevou a taxa de mortalidade e apresentou efeito prejudicial no desempenho e metabolismo. O elevado nível de nitrogénio não proteico é um dos fatores mais limitantes para a utilização da levedura de cana (BERTO, 1997), não possuindo valor nutricional, podendo influenciar no cálculo do nível de proteína bruta, uma vez que o valor de $\mathrm{N}$ x 6,25, provavelmente, superestima o conteúdo de proteína digestível (TACON \& COOKE, 1980).

A proteína da dieta é prioritariamente fornecida com o objetivo de ser utilizada para a síntese proteica nos tecidos, sendo que a eficiência da sua utilização está relacionada com a quantidade e também qualidade da proteína ingerida. A expansão da piscicultura tem levado a numerosos estudos no sentido de avaliar fontes alternativas de proteína na alimentação de peixes. Justifica-se, então, o seu estudo da utilização da levedura pela disponibilidade regional, seu elevado valor nutricional e menor custo em relação ao farelo de soja. O presente trabalho teve por objetivo avaliar os efeitos de diferentes níveis de inclusão de levedura seca (S. cerevisiae) "spray-dried", sobre o desempenho e eficiência económica de alevinos revertidos de tilápias do Nilo (Oreochromis nilotícus).

\section{MATERIAL E MÉTODOS}

O experimento foi realizado no Laboratório de Aquicultura da Universidade Estadual de Maringá (UEM), Paraná, durante o período de 4 outubro a 20 de dezembro de 1997 (48 dias). Foram utilizados 1200 alevinos de tilápia do Nilo com peso vivo médio de $0,13 \pm 0,01 \mathrm{~g}$, revertidos através do uso de $60 \mathrm{mg}$ do hormônio masculinizante 17-a-metiltestosterona, adicionado na proporção de $60 \mathrm{mg} / \mathrm{kg}$ de dieta, durante um período de 30 dias; obtidos da Estação de Piscicultura CODAPAR/UEM, distribuídos em 20 aquários de cimento amianto com capacidade individual de $1000 \mathrm{~L}$ (800L de volume útil), mantidos com água oriunda de poço artesiano da UEM, taxa de renovação de água de 15\%/dia e aeração através de bombas de ar comprimido. Semanalmente, era realizada a sifonagem para retirada dos resíduos.

Foram elaboradas seis rações com níveis de 0,$0 ; 7,8 ; 15,6 ; 23,4$ e $31,2 \%$ de inclusão de levedura desidratada (Tabela 1). As rações foram fornecidas na forma de pasta, parcelada em três vezes ao dia ( $8 \mathrm{~h} ; 12 \mathrm{~h}$ e $16 \mathrm{~h})$, na proporção de $8 \%$ sobre a biomassa de cada tanque, fornecidas em comedouros tipo calha, sendo o arraçoamento realizado na forma manual. A análise dos alimentos foi realizada no Laboratório de Análises de Alimentos do Departamento de Zootecnia da Universidade Estadual de Maringá de acordo com a metodologia descrita por SILVA (1990). 
Tabela 1 - Composição bromatológica e percentual das rações experimentais com e sem inclusão de levedura desidratatda (com base na matéria seca).

\begin{tabular}{|c|c|c|c|c|c|}
\hline \multirow[b]{2}{*}{ Ingredientes } & \multicolumn{5}{|c|}{ Níveis de inclusão de levedura desidratada $(\%)^{2}$} \\
\hline & 0,0 & 7,8 & 15,6 & 23,4 & 31,2 \\
\hline Milho moído & 41,39 & 39,81 & 35,43 & 35,58 & 33,29 \\
\hline Farelo de soja & 37,79 & 31,80 & 29,12 & 21,24 & 15,82 \\
\hline Farinha de peixe & 15,00 & 15,00 & 15,00 & 15,00 & 15,00 \\
\hline Calcário calcitico & 0,42 & 0,51 & 0,97 & 1,23 & 1,44 \\
\hline Fosfato bicálcico & 1,15 & 1,33 & 0,63 & 0,30 & 0,00 \\
\hline Óleo de soja & 3,00 & 2,50 & 2,00 & 2,00 & 2,00 \\
\hline Sal comum & 0,25 & 0,25 & 0,25 & 0,25 & 0,25 \\
\hline Premix min. e vitam ${ }^{3}$. & 1,00 & 1,00 & 1,00 & 1,00 & 1,00 \\
\hline Total & 100,00 & 100,00 & 100,00 & 100,00 & 100,00 \\
\hline $\operatorname{MS}(\%)$ & 91,15 & 90,17 & 92,11 & 91,16 & 92,05 \\
\hline $\mathrm{ED}(\mathrm{kcal} / \mathrm{kg})^{4}$ & 3275,56 & 3313,94 & 3308,67 & 3414,15 & 3465,33 \\
\hline PB (\%) & 32,73 & 32,85 & 32,41 & 32,39 & 32,30 \\
\hline $\mathrm{Ca}(\%)$ & 1,30 & 1,25 & 1,11 & 1,25 & 1,34 \\
\hline Ptotal (\%) & 1,11 & 0,96 & 0,92 & 1,05 & 0,98 \\
\hline
\end{tabular}

Não foram $(\mathrm{P}>0,05)$ dos níveis de inclusão de levedura desidratada sobre as variáveis de sobrevivência e de conversão alimentar aparente. $\mathrm{O}$ resultado de sobrevivência encontrado no presente estudo discorda dos valores encontrados por PADUA (1996), possivelmente em função do maior nível de inclusão utilizado por esse autor, de $39,8 \%$ para o pacu (Piaractus mesopotamicus), durante o período de 40 a $120 \mathrm{~g}$, nível em que foi encontrada elevada taxa de mortalidade em relação aos demais tratamentos com níveis inferiores de inclusão de levedura.

Com o aumento nos níveis de inclusão de levedura desidratada, foi observado efeito quadrático $(\mathrm{P}<0,05)$ sobre ganho de peso e custo em ração/kg de ganho de peso (Figuras $1 \mathrm{e}$

Para a determinação do custo em ração/kg de ganho de peso, utilizou-se a equação proposta por BELLAVER et al. (1985). Ao final do experimento, todos os animais de cada unidade experimental foram pesados $(\mathrm{g})$ e medidos $(\mathrm{cm})$ individualmente. A cada três dias, foram tomadas as medidas de $\mathrm{pH}$, condutividade elétrica $(\mu \mathrm{S} / \mathrm{cm})$ e oxigênio dissolvido $(\mathrm{mg} / \mathrm{L})$ da água. A temperatura da água foi medida diariamente às 8 e 17 horas.

O delineamento experimental utilizado foi o inteiramente casualizado com cinco tratamentos e quatro repetições, sendo a unidade experimental constituída por um tanque com 60 animais. Os dados obtidos foram submetidos às análises de variância e regressão polinomial, utilizando-se o programa SAEG (sistema para análises estatísticas e genéticas), desenvolvido por EUCLYDES (1983).

\section{RESULTADOS E DISCUSSÃO}

Na tabela 2, encontram-se os resultados médios de desempenho e custo em ração/kg de ganho de peso, em função da inclusão de níveis crescentes de levedura desidratada nas rações.
2). Os melhores valores estimados para essas variáveis foram obtidos com 14,03 e $14 \%$, respectivamente, de inclusão de levedura desidratada, o que correspondeu a uma participação de cerca de $17,41 \%$ da proteína bruta da

Tabela 2 - Valores médios de desempenho e custo em ração/kg de ganho de peso de alevinos revertidos de tilápias do Nilo, em função dos níveis de inclusão de levedura desidratada.

\begin{tabular}{lrrrrrr}
\hline & \multicolumn{6}{c}{ Níveis de inclusão de levedura desidratada (\%) } \\
\cline { 2 - 7 } Variáveis & \multicolumn{1}{c}{0,0} & 7,8 & 15,6 & 23,4 & 31,2 & $\mathrm{CV}(\%)$ \\
& & & & & & \\
& & & & & \\
PI (g) & 0,13 & 0,12 & 0,12 & 0,13 & 0,12 & 5,67 \\
PF (g) & 1,94 & 2,15 & 1,94 & 2,10 & 1,42 & 17,27 \\
GP (g)* & 1,81 & 2,03 & 1,82 & 1,97 & 1,30 & 10,18 \\
CAa & 1,87 & 1,97 & 2,03 & 1,73 & 2,43 & 12,80 \\
S (\%) & 97,30 & 98,33 & 98,33 & 98,74 & 97,08 & 2,05 \\
R \$/kg & 0,61 & 0,54 & 0,62 & 0,52 & 0,71 & 10,73 \\
ganho* & & & & & & \\
\hline
\end{tabular}

$\mathrm{PI}=$ peso inicial; $\mathrm{PF}=$ peso final; $\mathrm{GP}=$ ganho de peso; $\mathrm{CAa}=$ conversão alimentar aparente; $S=$ sobrevivência e $\mathrm{R} \$ / \mathrm{kg}$ ganho $=$ custo em ração/quilo de ganho de peso.

* Efeito quadrático $(\mathrm{P}<0,05)$ :

$\mathrm{GP}\left(\mathrm{Y}=1,7133+0,0477 \mathrm{X}-0,0017 \mathrm{X}^{2}\right.$

$\left.r^{2}=0.78\right) ; Y i\left(Y=0.6170-0.0112 X+0.0004 X^{2}: r^{2}=0.63\right)$. 


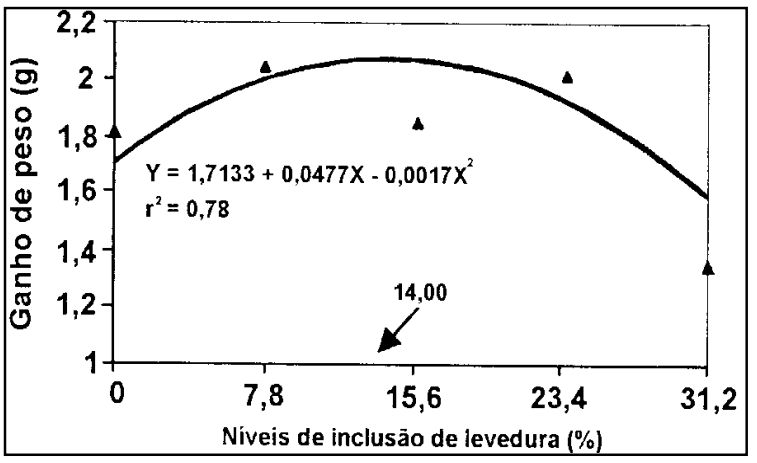

Figura 1 - Ganho de peso de alevinos revertidos de tilápias do Nilo, em função dos níveis de inclusão de levedura desidratada.

levedura desidratada, ou aproximandamente $27,21 \%$ de substituição da proteína do farelo de soja pela proteína da levedura desidratada.

Esses valores aproximam-se dos obtidos por DAVIES \& WAREHAM (1988), que encontraram uma redução progressiva no valor da ração com níveis acima de $15 \%$ de levedura para a tilápia (Oreochromis mossambicus). Os níveis de inclusão obtidos no presente trabalho são inferiores aos encontrados por RUMSEY et al. (1990) para a truta do lago, na qual o melhor crescimento foi observado com $50 \%$ de inclusão de levedura, e de PADUA (1996), que não observou efeito prejudicial sobre o desempenho e metabolismo do pacu durante o período de 40 a $120 \mathrm{~g}$ até o nível de $29,88 \%$ de inclusão desse alimento.

O melhor desempenho obtido com a inclusão de levedura seca, provavelmente, ocorreu pelo melhor balanceamento de nutrientes, destacando-se a associação dos aminoácidos, assim como da presença de vitaminas, principalmente do complexo B. Ainda que a levedura desidratada seja deficiente em alguns aminoácidos essenciais, principalmente sulfurados, a combinação com o farelo de soja e a farinha de peixe permitiu um melhor balanceamento dos mesmos.

A piora no ganho de peso, a partir do nível estimado, reforça a citação de PEZZATO (1995), de que altos níveis de inclusão de levedura leva a uma maior excreção de amônia. De acordo com BERTO (1997), um dos fatores mais limitantes para a utilização da levedura de cana é o elevado nível de nitrogénio não proteico, que corresponde a 20 a $30 \%$ do nitrogênio total, que é representado principalmente por ácidos nucléicos, de 8 a $12 \%$ do nitrogénio total (BUTOLO, 1997), sendo que os resultados obtidos

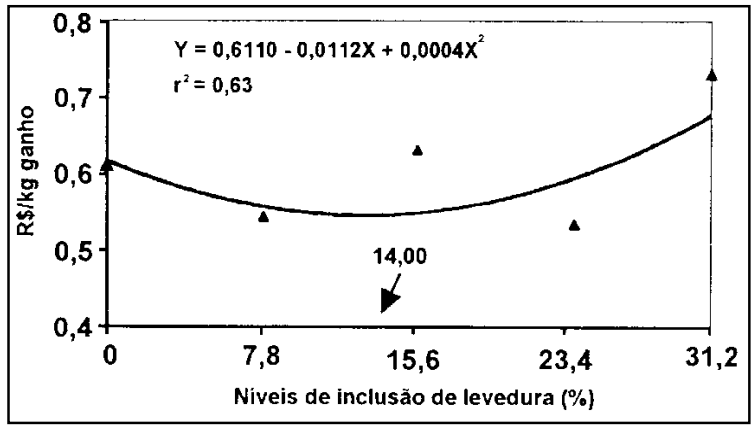

Figura 2 - Custo em ração/kg de ganho de peso ( $\mathrm{R} \$ \mathrm{~kg}$ ganho), de alevinos revertidos de tilápias do Nilo, em função dos níveis de inclusão de levedura desidratada.

nesse trabalho concordam com esse último autor quanto ao nível de inclusão, em valores próximos a $10 \%$ para animais não-ruminantes.

Os resultados reforçam as citações de TACON \& COOKE (1980), de que elevada taxa de inclusão de ácido nucléico resulta em piora no desempenho, sugerindo que não possuem valor nutricional e, além disso, podem influenciar no cálculo do nível de proteína bruta, uma vez que foi utilizado o valor de $\mathrm{N}$ x 6,25, o que, provavelmente, superestimou o conteúdo de proteína digestível nas rações, sendo esse efeito negativo mais pronunciado no nível de maior inclusão de levedura desidratada, de $31,2 \%$.

$\mathrm{Na}$ tabela 3, encontram-se os valores médios da temperatura, oxigênio dissolvido, $\mathrm{pH}$ e condutividade elétrica da água dos tanques. A temperatura média da água dos tanques experimentais foi inferior àquela exigida para 0 máximo desenvolvimento dessa espécie, de 25 a $30^{\circ} \mathrm{C}$, sendo observado um maior consumo das $12 \mathrm{~h}$ às $18 \mathrm{~h}$, período em que foram concentradas as refeições. Por outro lado, os demais parâmetros analisados encontram-se dentro da faixa adequada para se obter um bom desempenho produtivo dessa espécie (EGNA \& BOYD, 1997).

Apesar da levedura desidratada ser considerada como um ingrediente com potencial para reduzir a qualidade da água, não foram observados efeitos $(\mathrm{P}>0,05)$ dos diferentes níveis de inclusão de levedura seca sobre os parâmetros avaliados. O fato
Tabela 3 - Valores médios de temperatura, oxigênio dissolvido, pH c condutividade elétrica da água dos tanques, em função dos níveis de inclusão de levedura desidratada.

\begin{tabular}{lrrrrrr}
\hline & \multicolumn{6}{c}{ Níveis de inclusão de levedura desidratada (\%) } \\
\cline { 2 - 7 } Variáveis & \multicolumn{1}{c}{0,0} & 7,8 & 15,6 & 23,4 & 31,2 & CV (\%) \\
\hline Temperatura (cC) & 23,34 & 23,25 & 23,27 & 23,30 & 23,32 & 8,16 \\
Oxigênio dissolvido (mgL) & 4,32 & 4,67 & 4,43 & 4,28 & 4,26 & 5,80 \\
pH & 8,73 & 8,71 & 8,61 & 8,64 & 8,67 & 2,35 \\
Condutividade elétrica $(\mu \mathrm{S} / \mathrm{cm})$ & 17,11 & 18,25 & 17,14 & 18,11 & 18,16 & 4,11 \\
\hline
\end{tabular}


de as rações terem sido fornecidas na forma de pasta e em comedouros pode ter contribuído para a menor lixiviação dos nutrientes e, conseqüentemente, manutenção na qualidade da água, uma vez que esse ingrediente demonstrou ser um excelente agente para auxiliar a aglutinação, pelo tamanho da sua partícula, menor ou igual a $500 \mu$ n e/ou pela presença de carboidratos.

Pelos resultados obtidos, a levedura desidratada de cana-de-açúcar demonstrou ser um excelente alimento para alevinos revertidos de tilápias do Nilo, quer seja sobre o desempenho e também na redução no custo de produção, sendo necessário observar os níveis de inclusão, principalmente pela incorporação de bases nitrogenadas, que podem levar a uma redução no desempenho, o que também dificulta o cálculo do nível proteico verdadeiro das rações, uma vez que não se sabe o valor nutricional dessa fonte de nitrogénio.

\section{CONCLUSÕES}

Nas condições em que foi realizado o presente trabalho, conclui-se que a levedura seca (Saccharomyces cerevisiae) "spray-dried" pode ser incluída em até $14 \%$, em rações para alevinos revertidos de tilápias do Nilo (Oreochromis niloticus).

\section{AGRADECIMENTOS}

Aos funcionários e estagiários do Laboratório de Aquicultura do Centro de Ciências Biológicas, pelo apoio nas diversas etapas desta pesquisa.

\section{REFERÊNCIAS BIBLIOGRÁFICAS}

ALVES, L.M., PEZZATO, L.E., GUEREIRO NETO, A.C., $\boldsymbol{e} \boldsymbol{t}$ al Avaliação de níveis crescentes de levedura seca Saccharomyces cerevisiae, de vinhaça incorporados às rações de tilápias-do-Nilo $\boldsymbol{O}$. niloticus. In: SIMPÓSIO LATINO AMERICANO DE AQUICULTURA, 6, SIMPÓSIO BRASILEIRO DE AQUICULTURA, 5, 1988 Florianópolis,SC. Anais.... Florianópolis : SIMBRAq, CBNA, 1988. p.211.

BELLAVER, C., FIALHO, E.T, FROTAS, J.F.S. Radícula de malte na alimentação de suínos em crescimento e terminação. Pesquisa Agropecuária Brasileira, v.20, n.8, p.969974,1985 .

BERTO, D. A. Uso da levedura desidratada na alimentação de suínos. In: SIMPÓSIO SOBRE TECNOLOGIA DA PRODUÇÃO E UTILIZAÇÃO DA LEVEDURA DESIDRATADA NA ALIMENTAÇÃO ANIMAL, 1997, Campinas - SP. Anais... Campinas : CBNA, 1997. p.85-106.

BUTOLO, J.E. Uso da levedura desidratada na alimentação de aves. In : SIMPÓSIO SOBRE TECNOLOGIA DA PRODUÇÃO E UTILIZAÇÃO DA LEVEDURA
DESIDRATADA NA ALIMENTAÇÃO ANIMAL, 1997, Campinas, SP. Anais... Campinas : CBNA, 1997. p.51-84.

DAVIES, S.J., WAREHAM, H. A. A preliminary evaluation of na industrial single cell protein in practical diets for tilapia (Oreochromis mossambicus, Peters). Aquaculture, Amsterdam, v.73, p.189-199,1988.

DESMONTS, R. Utilização da levedura na alimentação de crianças. Pediatria Prática, v.39, n.7, p.7-18,1968.

EGNA, H. S., BOYD, C. E. Dynamics of pond aquaculture. Boca Raton :CRC, 1997. 318p.

EUCLYDES, F.R. Manual de utilização do programa SAEG (Sistema para Análise Estatísticas e Genéticas). Viçosa: UFV, 1983.59p.

GHIRALDINI, J.A., ROSSELL, C.E. Caracterização e qualidade da levedura desidratada para a alimentação animal, In: SIMPÓSIO SOBRE TECNOLOGIA DA PRODUÇÃO E UTILIZAÇÃO DA LEVEDURA DESIDRATADA NA ALIMENŢAÇÃO ANIMAL, 1997, Campinas, SP. Anais... Campinas: CBNA, 1997. p.27-50.

KRONKA, R.N., ARCADEPANI, D., RAMOS LA., $\boldsymbol{e}$ t al. Utilização da levedura seca (Saccharomyces cerevisiae) de destilarias de álcool de cana-de-açúcar e farelo de arroz na alimentação de suínos nas fases inicial, crescimento e terminação (experimento 2). Arquivos de Veterinária, v.7, n.1.p.64-77,1991.

LANDELL, L.C., KRONKA, R.N., THOMAZ, M.C., $\boldsymbol{e t}$ al. Utilização da levedura de centrifugação de vinhaça (Saccharomyces cerevisiae) como fonte proteica para leitões na fase inicial (10 a $30 \mathrm{~kg}$ PV). Revista da Sociedade Brasileira de Zootecnia, v.23, n.2, p.283-291,1994.

MIYADA, V.S, LAVORENTI, A. Uso da levedura seca (Saccharomyces cerevisiae) de destilarias de álcool de canade-açúcar na alimentação de suínos em crescimento e acabamento. Revista da Sociedade Brasileira de Zootecnia, v.8, n.3, p.497-515,1979.

NRC - National Research Council. Nutritional requirements of tisnes. Washington : Academic. 1993. 114p.

PADUA, D.M.C. Utilização da levedura alcoólica (Saccharomyces cerevisiae) como fonte proteica na alimentação de juvenis de pacu (Piaractus mesopotamicus), PISCES, TELEOSTEI): aspectos metabóBcos e de desempenho produtivo. Jaboticabal, SP, 1996. 120p. Dissertação (Mestrado em Aquicultura) Universidade Estadual Paulista - UNESP, Centro de Aquicultura - CAUNESP, Jaboticabal, 1996.

PERRY, R.H., GREEN, D.W, MALONEY, J.O. Perry's engineers' handbook 6ed. Toldo: McGraw-Hill, 1984. 420p.

PEZZATO L.E., TAMBURO, M. E., PEZZATO, A.C., $\boldsymbol{e} \boldsymbol{t}$ al Levedura (Saccharomyces cerevisiae) de álcool de cana-deaçúcar como fonte proteica da alimentação de carpas (Cyprinus carpia), to: REUNIÃO DA SOCIEDADE BRASILEIRA DE ZOTECNIA, 19, 1982, Piracicaba. Anais... Piracicaba: SBZ, 1982. p.467.

PEZZATO L.E., TAMBURO, M. E., PEZZATO, A.C., et al. Levedura (Saccharomyces cerevisiae) de álcool de cana-deaçúcar como fonte protéica da laimentação de carpas (Cyprinus carpio). In: REUNIÃO DA SOCIEDADE BRASILEIRA DE ZOOTECNIA, 19, 1982, Piracicaba. Anais... Piracicaba : SBZ, 1982. P.467. 
PEZZATO, L.E. Alimentos convencionais e não-convencionais disponíveis para indústria da nutrição de peixes no Brasil. In: SIMPÓSIO INTERNACIONAL SOBRE NUTRIÇÃO DE PEIXES E CRUSTÁCEOS, 1995, Campos do Jordão. Anais... Campos de Jordão: CBNA, 1995. p.34-52.

RUMSEY. G. L., HUGHES, S.G., KINSELLA, J.L. Use of dietary yeast Saccharomyces cerevisiae nitrogen by lake trout. Journal of World Aquaculture Society, v.21, n.3, p.205-209, 1990.
SILVA, S.S. Análise de alimentos (métodos químicos e biológicos). 2 ed. Viçosa: UFV, 1990.166p.

SINTAYEHU, A., MATHIES, E., MEYER-BURFDORFF, K.H., et al. Apparent digesdbilies and growth experiments with tilapia (Oreochromis niloticus) fed soybean meal, cottonseed meal and sunflower seed meal. Journal Applied Ichthyology, v.12, n.2, p.125-130,1996.

TACON, A.G.J.,COOKE, D.J. The nutritional value of dietary nucleic acids to trout. Nut Repr Int, v.22, p. 631

Ciência Rural, v. 30, n. 4, 2000. 